\title{
RESISTANCE-DEPENDENT FIELD EFFECT ON THE RADIATION BEHAVIOR OF MOS CAPACITORS EXAMINED BY INSTANTANEOUS-TERMINAL-VOLTAGE TECHNIQUE
}

\author{
JENN-Gwo HwU and SHYH-LIANG Fu \\ Department of Electrical Engineering, National Taiwan University, Taipei, Taiwan, Republic of China
}

(Received 8 August 1988; accepted in revised form 9 August 1989)

\begin{abstract}
Using an instantaneous-terminal-voltage (ITV) measurement technique, the variation of the oxide field in an MOS capacitor irradiated by $\mathrm{X}$-rays without bias is examined. It is shown that the oxide field during irradiation depends on the device leakage resistance which can be estimated by the newly reported charge-then-decay method, and the flat-band voltage shift due to irradiation is consistent with the field so found. Interestingly, it is found that the device leakage resistance during irradiation for an $\operatorname{MOS}(p)$ capacitor is generally smaller than that for an $\operatorname{MOS}(n)$ capacitor. The different radiation sensitivities between an $\operatorname{MOS}(p)$ and an $\operatorname{MOS}(n)$ capacitor should account primarily for the dopantdependent oxide resistances found in this work instead of the initial work function differences alone. An equivalent circuit is given to describe the dependence of the oxide field during irradiation on the device resistance, and to explain the observations.
\end{abstract}

Keywords: Resistance effect, radiation, MOS capacitor.

\section{INTRODUCTION}

The effect of radiation on MOS devices has received much attention recently since it can seriously degrade the devices' performance [1]. Much work has been done about the dependence of the radiation effect on the bias $[2,3]$. The bias determines the intensity of the oxide field in an MOS device during X-ray irradiation, so that the radiation result, which depends on the separation of the radiation-induced electrons and holes within the oxide layer, is controllable by the bias. In these studies, the oxide fields are clearly defined since the devices' biases are supplied by the known voltage sources. However, there are other works about the radiation behavior of certain specified samples which are irradiated without biases, i.e. with gates floating during irradiations [4-6]. The built-up oxide fields for these samples during irradiations are surely important to the radiation results. Unfortunately, there is still a lack of discussion about the oxide field under such circumstance. This is probably due to the difficulty of the experimental oxide field measurement. In this work, we propose a method to directly observe the field variation for an MOS capacitor irradiated by X-rays without bias. This is different from the conventional methods for examining the transient radiation behavior $[2,3,7]$. Briefly, the device under test is shunted by a high input-impedance electrometer (Keithley 617). When the sample receives an X-ray irradiation, its instantaneous-terminal-voltage (ITV) can be detected. Based on the ITV technique, some significant relations between radiation sensitivity and sample preparation are found.

It is known that samples, which are prepared the same way, may yield different radiation results due to many effects, e.g. the stress [4]. These effects may be difficult to distinguish before irradiation. However, if the samples are examined by the newly reported charge-then-decay method [8], one can easily distinguish their device resistances. The charge-then-decay method is briefly described as follows. The sample is initially charged with a certain voltage. When the charging source is removed from the sample, the charges on the metal gate (or the substrate) are not lost quickly due to the large value of the $R C$ time constant. From the voltage read by an electrometer, one can estimate the $R C$ time constant. If the value of $C$ is known, the device leakage resistance is then found. Based on the ITV technique proposed in this work, it is found that the device leakage resistance is indeed important to the oxide field during irradiation, and consequently the radiation result. Interestingly, it is observed that the oxide field for an $\operatorname{MOS}(p)$ capacitor is generally larger than that for an $\operatorname{MOS}(n)$ one during irradiation. This phenomenon mainly accounts for the dopant-dependent oxide resistance rather than the initial work function difference $\phi_{M S}$ alone [6]. The explanation is given by an equivalent circuit describing the dependence of the oxide field on the device resistance during irradiation. Finally, the transient peak appearance just as the X-ray is turned on, the slowly increasing behavior of the voltage read during irradiation, and the voltage drop occurring 
just as the $X$-ray is turned off during the ITV measurement, are explained.

\section{EXPERIMENTAL}

$p$ and $n$ MOS capacitors were used in this work. The samples with oxide thicknesses of $960 \AA$ were prepared by wet oxidation, and those with thicknesses of 660 and $350 \AA$ by dry oxidation. Aluminum was used as the material of the metal gate and substrate contact, and the gate area was $3.6 \times 10^{-3} \mathrm{~cm}^{2}$. All the samples were standard IC packaged (24 pins). An electrometer (Keithley 617), with an input resistance of $10^{14} \mathrm{ohm}$ shunted by an input capacitance of $20 \mathrm{pF}$, was used to monitor the MOS terminal voltage.

To start with, three packaged $\operatorname{MOS}(p)$ capacitors with oxide thickness of $960 \AA$ were examined. These samples were prepared the same way. Their device leakage resistances, $r$ were characterized by the charge-then-decay method as shown in Fig. I(a). The sample under test was charged to $-5 \mathrm{~V}$ first, then the charging source was removed from the MOS capacitor. The voltages read by the electrometer are shown in Fig. 1(b). From the terminal voltages, the $V$, s, read at $t=5 \mathrm{~min}$, one can estimate their $R C$ time constants based on a simple $R C$ decay equation. The corresponding $R C$ time constants for samples 1,2 and 3 are about 6450,960 and $250 \mathrm{~s}$, respectively. According to the known capacitances of the MOS capacitor (about $100 \mathrm{pF}$ ) and the electrometer (about $20 \mathrm{pF}$ ), the $r s$ for samples 1,2 and 3 are found to be about $5 \times 10^{13}, 8 \times 10^{12}$, and $2 \times 10^{12}$ ohms, respectively. They are quite different from each other.

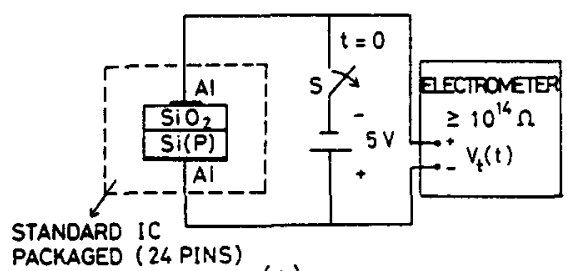

(a)

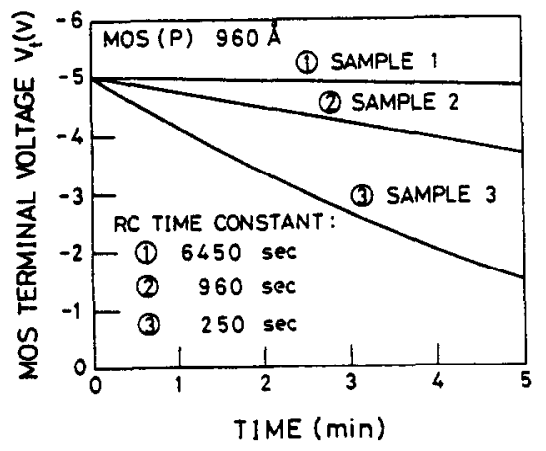

(b)

Fig. 1. (a) The schematic diagram of the charge-then-decay measurement system for MOS capacitors. (b) MOS terminal voltage $V$, vs time for three $\operatorname{MOS}(p)$ capacitors decaying from $-5 \mathrm{~V}$.
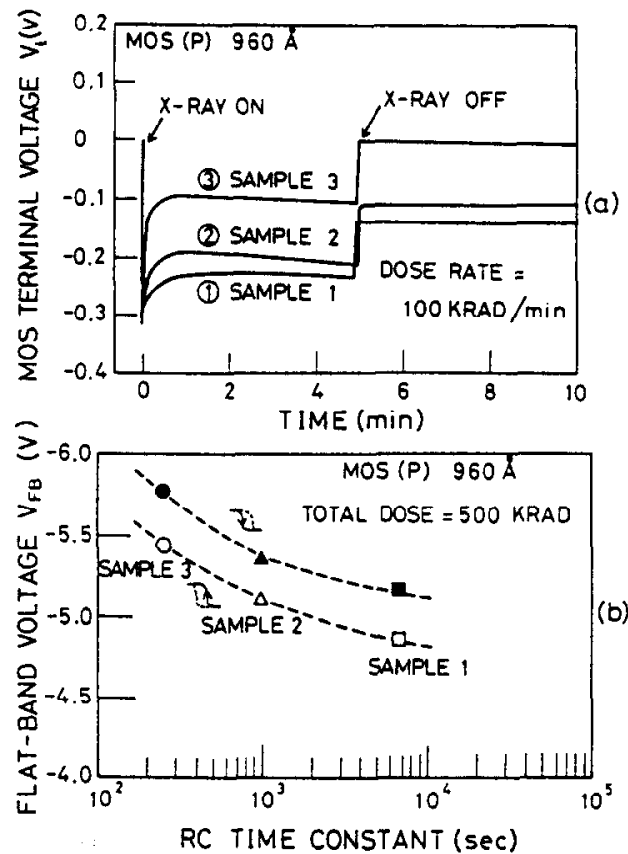

Fig. 2. (a) MOS terminal voltage $V$, vs time for three $\operatorname{MOS}(p)$ capacitors during and after $\mathrm{X}$-ray irradiation with the same dose rate of $100 \mathrm{krad} \mathrm{min}^{-1}$ for $5 \mathrm{~min}$. (b) Flatband voltage $V_{F B}$ vs $R C$ time constant for these capacitors after irradiation.

Next, these samples were irradiated by $\mathrm{X}$-rays with the same dose rate of $100 \mathrm{krad} \mathrm{min}^{-1}$ for $5 \mathrm{~min}$, individually. Their instantaneous $V, s$ vs time during and after irradiations are shown in Fig. 2(a). Note that all the $V$, values are negative. $V$, reaches a peak value within a short period after the $X$-ray is on, then it is almost in steady state except for slowly increasing with time during irradiation. Let $V_{\text {is }}$ be the steadystate terminal voltage read during irradiation. It is observed that the sample having the largest $r$, e.g. sample 1, has the largest $V_{s s}$. Also, note that all the $V_{t s}$ s, i.e. $-0.23,-0.2,-0.1 \mathrm{~V}$ for samples 1,2 and

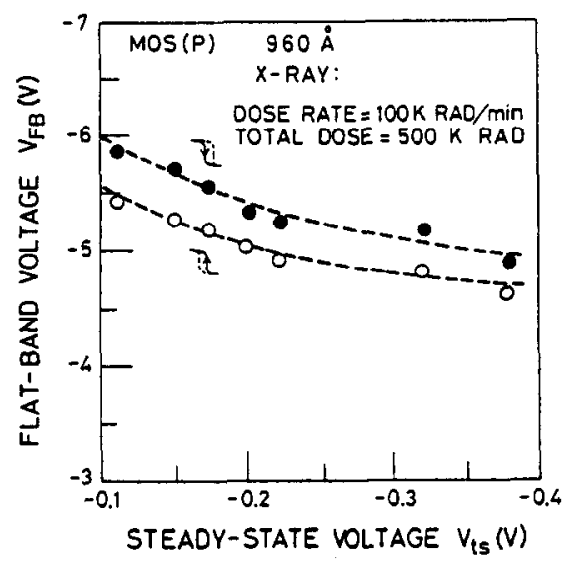

Fig. 3. Flat-band voltage $V_{F B}$ vs steady-state voltage $V_{t}$ for various $\operatorname{MOS}(p)$ capacitors having different device leakage resistances $r$. 

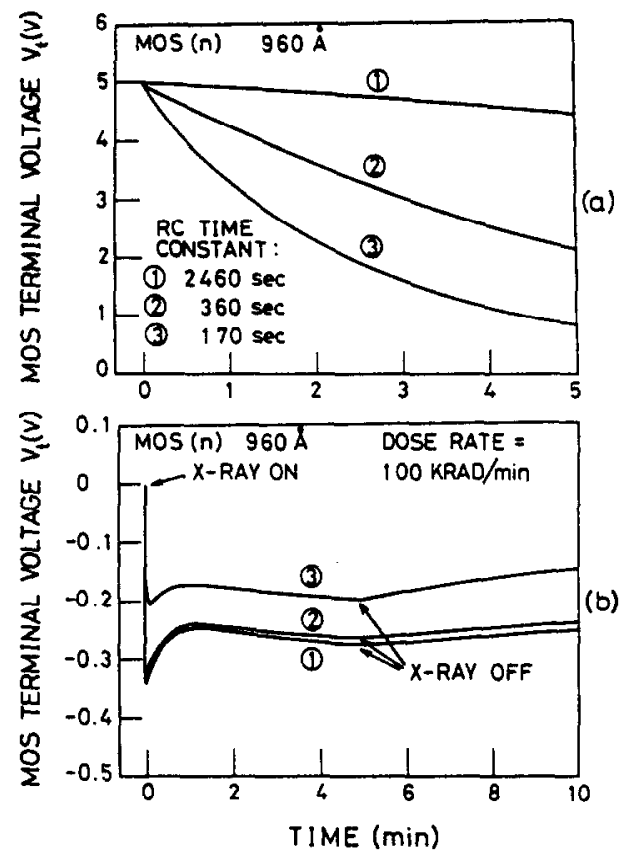

Fig. 4. (a) MOS terminal voltage $V$, vs time for three $\operatorname{MOS}(n)$ capacitors decaying from $+5 V$. (b) $V$, vs time for these capacitors during and after $\mathrm{X}$-ray irradiation.

3, respectively, are much smaller than the $\phi_{M S}$ of an $\operatorname{MOS}(p)$ capacitor, i.e. about $-0.88 \mathrm{~V}$ in this work. Furthermore, when the $\mathrm{X}$-ray is off, all the $V_{t} \mathrm{~s}$ drop sharply first and then decay slowly with time. Figure 2(b) shows the flat-band voltages $V_{F B} \mathrm{~s}$ of these samples after irradiation vs the $R C$ time constants; because every $C-V$ curve (swept forward and backward subsequently) after irradiation shows a hysteresis loop due to the increase of slow trap states, two $V_{F B} s$ are obtained from one sample. It can be seen from this figure that the larger the $R C$ time constant, the smaller the $V_{F B}$. Thus, the radiation result depends on $r$. Figure 3 shows the relationship between $V_{F B}$ and $V_{t s}$ for various samples prepared the same way as mentioned above. It is clear from this figure that $V_{t s}$, which is intimately related to $r$, is important for radiation sensitivity.

Similarly, three $\operatorname{MOS}(n)$ capacitors with the same oxide thickness of $960 \AA$ as mentioned above, were also examined for comparison. Figure 4(a) shows the decays of $V$, vs time for these $\operatorname{MOS}(n)$ capacitors. The $V_{s} s$ were measured under the same procedure as mentioned in Fig. 1(a) except that the charging voltages were changed to $+5 \mathrm{~V}$. Their $R C$ time constants, i.e. 2460,360 and $170 \mathrm{~s}$, are clearly different from each other. Figure $4(\mathrm{~b})$ shows $V$, vs time for these samples during and after irradiation. Most of the phenomena mentioned in Fig. 2(a) are also observed in this figure, except that there are neglectable voltage drops just as the $\mathrm{X}$-ray is turned off and the $V_{t s}$ s, i.e. $-0.26,-0.25$ and $-0.19 \mathrm{~V}$ for curves 1,2 and 3 , respectively, are closer to the $\phi_{M S}$ of an
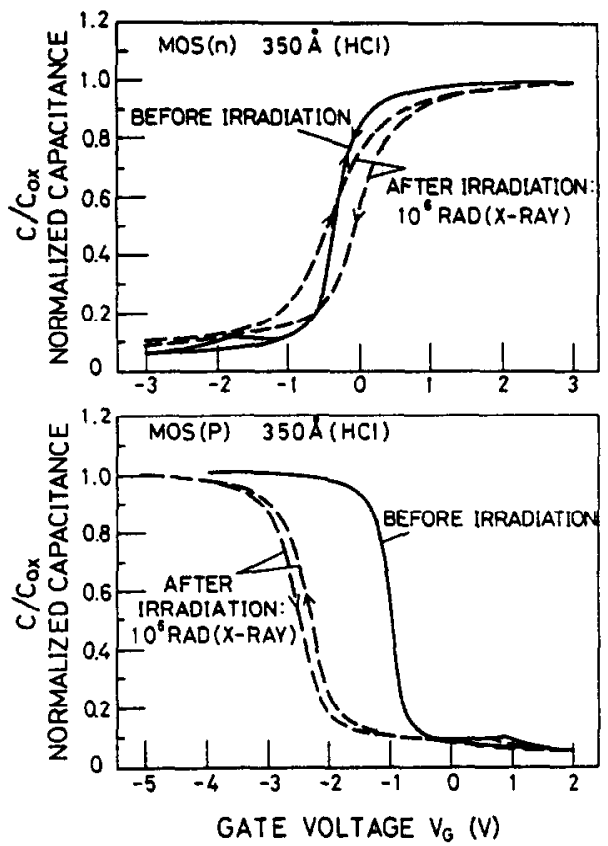

Fig. 5. Typical $C-V$ curves of an $\operatorname{MOS}(n)$ and an $\operatorname{MOS}(p)$ capacitor before and after irradiation.

$\operatorname{MOS}(n)$ capacitor, i.e. about $-0.3 \mathrm{~V}$ in this work, than those observed for the $\operatorname{MOS}(p)$ capacitors. Detailed descriptions will be given in a later section. Figure 5 shows typical $C-V$ curves of an $\operatorname{MOS}(n)$ and an $\operatorname{MOS}(p)$ capacitor before and after irradiation. The oxide thickness for these samples was $350 \AA$. Both of them were irradiated without biases as mentioned above. It is to be noted that the radiation effect for the $\operatorname{MOS}(p)$ capacitor is more serious than that for the $\operatorname{MOS}(n)$ capacitor.

Since $r$ is important for radiation sensitivity, one can intentionally introduce an internal resistance $R$ to the device for a clearer understanding. Five $\operatorname{MOS}(p)$ capacitors shunted by external resistors with different resistances $R$ of $10^{4}, 10^{5}, 10^{6}, 10^{7}$ and $10^{8}$ ohms, respectively, were examined. It is to be noted that these external resistors were suitably shunted so that they were not irradiated, namely, their resistances were constant during irradiation. The $V_{t} \mathrm{~s}$ vs time for these samples during and after irradiation are shown in Fig. 6. It is also to be noted that the curve for $R=\infty$ in this figure was obtained from a sample without external resistance. Its $V_{t s}$ is the largest one among all of them. When the X-ray is off, $V$, for this curve decays very slowly. However, when $R$ is added, then $V_{1 s}$ decreases significantly. Obviously, the smaller is the $R$ value, the smaller is $V_{v s}$. The data for $R=10^{4}$ and $10^{5}$ ohms are not shown in this figure since they are beyond the resolution of the measurement, i.e. $\leqslant 10^{-5} \mathrm{~V}$. Also note that all the $V_{t} \mathrm{~s}$ for the samples having external resistors decay to zero quickly when the $\mathrm{X}$-rays are turned off. This is due mainly to a significant decrease of the $R C$ time constant when $R\left(\leqslant 10^{8} \mathrm{ohm}\right)$ is added. 


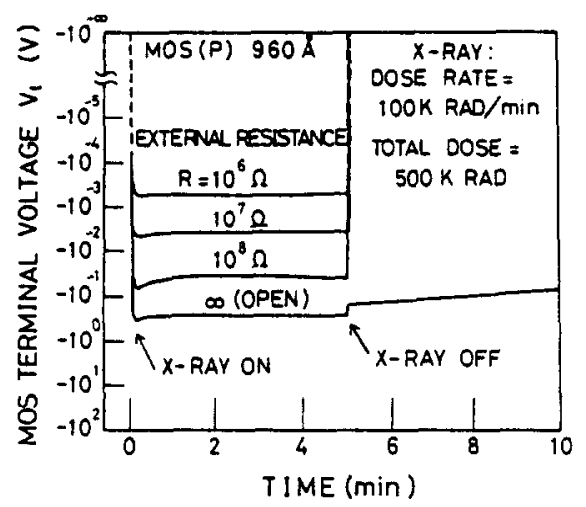

Fig. 6. MOS terminal voltage $V$, vs time for various $\operatorname{MOS}(p)$ capacitors having external resistances, $R$, during and after X-ray irradiation.

Figure 7 (a) shows the $V_{F B} s$ of these samples after irradiation vs $R$. Clearly, the smaller is the $R$, the larger is $V_{F B}$. The data obtained from a sample with $R=0$, i.e. short circuited, is also shown in this figure for comparison. Its $V_{F B}$ is the largest one among all of them. That is to say, a sample which is shortcircuited during irradiation should exhibit a higher sensitivity to irradiation than that which is opencircuit. Figure 7(b) shows the relationship between $V_{t s}$ and $R$ according to the data shown in Fig. 6 . It is strongly indicated from these figures that the value of $R$ determines the value of $V_{t s}$, which is intimately related to the oxide field during irradiation, and consequently the radiation effect.

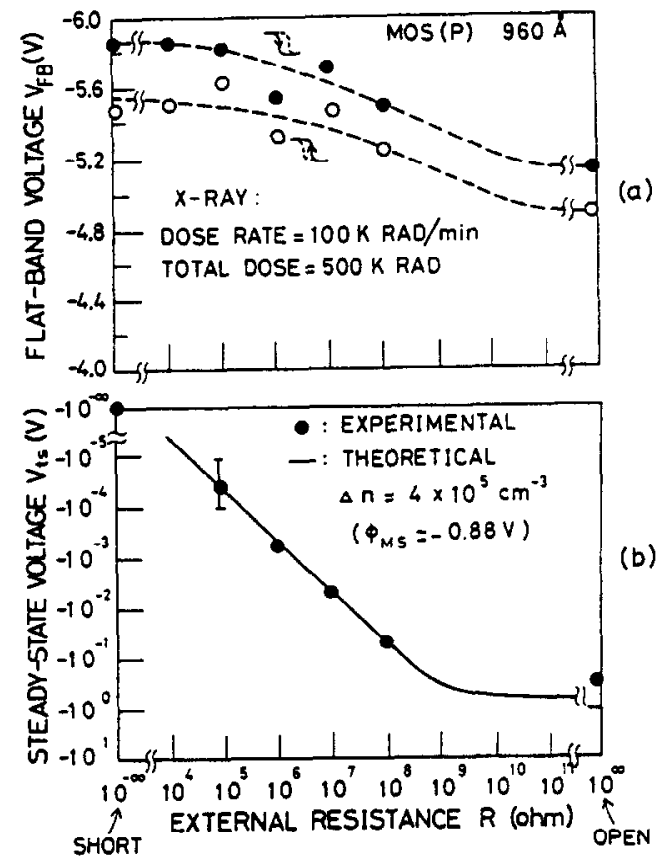

Fig. 7. (a) Flat-band voltage $V_{F B}$ vs external resistance $R$ for the capacitors of Fig. 6. (b) The experimental and theoretical relationships between steady-state voltage $V_{t s}$ and $R$ for these capacitors.

\section{EQUIVALENT CIRCUIT}

For an $\operatorname{MOS}(p)$ capacitor having a device leakage resistance of $r$, its energy band diagram under steadystate open-circuit condition can be represented by the schematic diagram shown in Fig. 8(a). Since the metal-semiconductor work function difference $\phi_{M S}$ is generally not equal to zero, i.e. about $-0.88 \mathrm{~V}$ in this work, the initial bulk oxide field $E_{1}$ under steady-state conditions can be expressed as

$$
E_{i} \cong \frac{\phi_{M S}}{d_{\mathrm{ox}}}
$$

where $d_{o x}$ is the oxide thickness. It is noted that the oxide charges near the $\mathrm{SiO}_{2} / \mathrm{Si}$ interface introduce image charges mainly in the silicon side but not in the metal side [9], so that the electric field introduced by these charges is mainly at the $\mathrm{SiO}_{2} / \mathrm{Si}$ interface and not at the bulk oxide. In this work, an electrometer and an external resistor (with a resistance of $R$ ), which were not irradiated by $\mathrm{X}$-rays during irradiation, were shunted to the MOS capacitor as indicated by the dotted lines in Fig. 8(b). When the MOS capacitor receives an $X$-ray irradiation, the instantaneous net radiation-induced electrons $\Delta n(t)$ and holes $\Delta p(t)$ at time $t$ will drift towards the metal gate and the substrate, respectively, according to the instantaneous bulk oxide field $E(t)$ as shown in Fig. 8(b). Therefore, the metal gate accumulates some

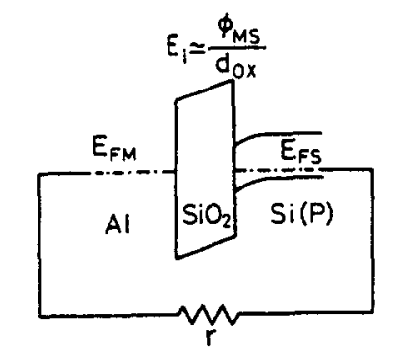

(DEVICE LEAKAGE RESISTANCE)

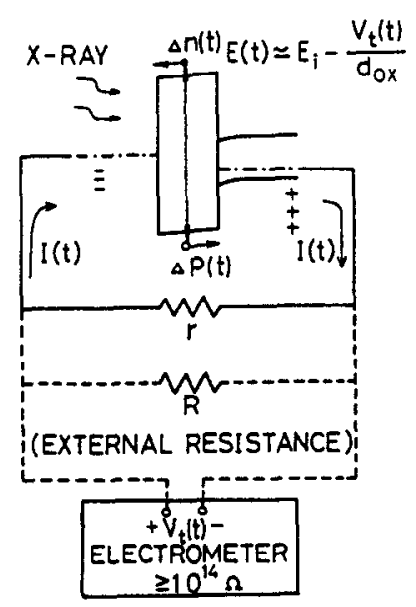

(b)

Fig. 8. The schematic diagrams of MOS capacitors (a) under open circuit steady-state condition and (b) under $\mathrm{X}$-ray irradiation. 
of the negative charges, whereas the substrate accumulates some of the positive charges. This separation of the electrons and holes during irradiation also occurs in an $\operatorname{MOS}(n)$ capacitor since the value of $\phi_{M S}$ for an $\operatorname{MOS}(n)$ capacitor is also negative, i.e. about $-0.3 \mathrm{~V}$ in this work. Therefore, it is expected that, for both the $\operatorname{MOS}(p)$ and the $\operatorname{MOS}(n)$ capacitors, the values of the instantaneous $V_{t} s$ due to irradiation are all negative as observed in Figs 2(a), 4(b) and 6.

Since the MOS capacitor during irradiation consists of a resistance, i.e. $r$ or $R$, the instantaneous current flowing through the device during irradiation at time $t$, i.e. $I(t)$, should not be zero; because the mobility of the hole $\mu_{p}$ is much smaller than the mobility of the electron $\mu_{n}$, i.e. $\mu_{n} \cong 20 \mathrm{~cm}^{2} \mathrm{~V}^{-1} \mathrm{~s}^{-1}$ at room temperature in the oxide layer [10], the $I(t)$ can be approximately expressed as

$$
I(t) \cong A q \Delta n(t) \mu_{n} E(t) .
$$

where $A$ is the area of the MOS capacitor. When the $V_{1}(t)$ is built-up at time $t$ due to the accumulation of the electrons at the metal gate and the holes at the substrate, as mentioned above, the $E(t)$ is reduced and can be approximately expressed as

$$
E(t) \cong E_{i}-\frac{V_{t}(t)}{d_{0 x}} .
$$

In the experiment when $R\left(\leqslant 10^{8} \mathrm{ohm}\right)$ is added, $r$ (which is on the order of $10^{9} \mathrm{ohm}$ during irradiation as discussed later) can be neglected. Thus,

$$
V(t) \cong I(t) R
$$

From eqns (2)-(4), one can obtain the following equations.

$$
\begin{gathered}
V(t) \cong \frac{A q \Delta n(t) \mu_{n} E_{i}}{\frac{1}{R}+\frac{A q \Delta n(t) \mu_{n}}{d_{\mathrm{ox}}}} \\
E(t) \cong \frac{E_{i}}{1+\frac{A q \Delta n(t) \mu_{n} R}{d_{\mathrm{ox}}}} .
\end{gathered}
$$

As can be seen from eqn (5), $V_{t}(t)$ can be strongly affected by $R$. The theoretical curve shown in Fig. 7(b) results from eqn (5) under steady-state condition with known parameters of $A=3.6 \times 10^{-3} \mathrm{~cm}^{2}, \mu_{n}=20 \mathrm{~cm}^{2} \mathrm{~V}^{-1} \mathrm{~s}^{-1}, d_{\mathrm{ox}}=960 \AA$, $\phi_{M S}=0.88 \mathrm{~V}$, and a fitted parameter of $\Delta n=4 \times 10^{3} \mathrm{~cm}^{-3}$. They fit the experimental data quite well except that the $V_{r s}$ for $R=\infty$ is much smaller than the ideal value, i.e. $V_{1 s}=\phi_{M S}=0.88 \mathrm{~V}$ according to eqn (5). That is to say $r$ can no longer be neglected when $R$ is absent.

According to eqn (6), it is to be noted that the smaller $R$, the larger $E(t)$. Thus, the separation of $\Delta n(t)$ and $\Delta p(t)$ is strong for the MOS capacitor with

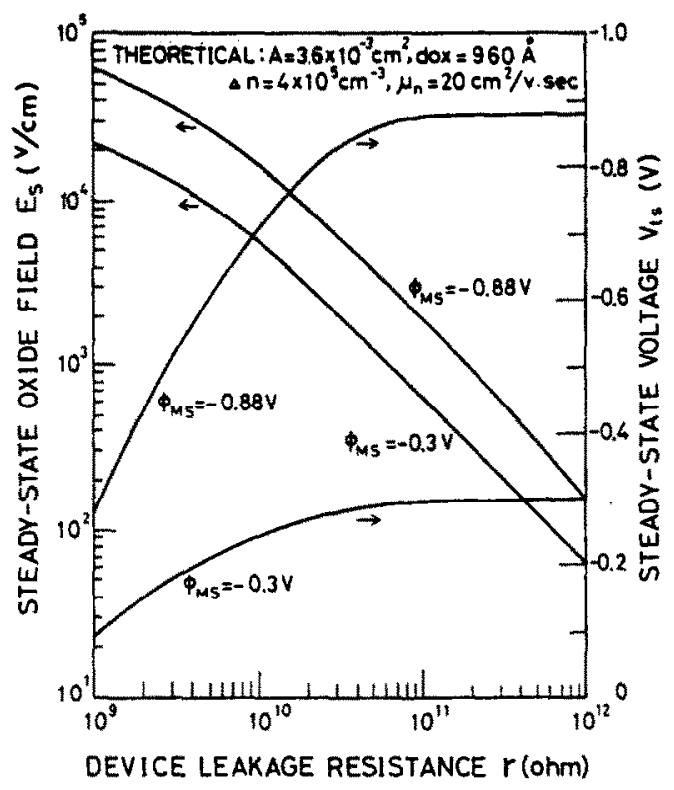

Fig. 9. The theoretical curves of steady-state oxide field $E_{s}$ and steady-state voltage, $V_{s s}$ vs device leakage resistance, $r$, for $\operatorname{MOS}(p)$ and $\operatorname{MOS}(n)$ capacitors under X-ray irradiation.

a small $R$. Under such circumstances, it is expected that the $V_{F B}$ after irradiation is large. This explains the relationship between $V_{F B}, R$ (or $r$ ), and $V_{i s}$ as observed in Figs 2(b), 3 and 7. Figure 9 shows the theoretical curves of $V_{t s}$ and steady-state oxide field $E_{s}$ vs $r$ according to eqns (5) and (6) for both the $\operatorname{MOS}(p)$ and $\operatorname{MOS}(n)$ capacitors with the parameters as mentioned above. It is noted that the $R$ in eqns (5) and (6) should be replaced by the $r$ when the $R$ is absent. Also note that although $r$ for a packaged sample before irradiation is generally greater than $10^{12} \mathrm{ohm}$, it may be reduced to a certain extent during irradiation. By comparing the data obtained from the open-circuit sample, i.e. $R=\infty$, and the theoretical curve as shown in Fig. 7(b), it is clear that the $r$ during irradiation for an $\operatorname{MOS}(p)$ capacitor is of the order of $10^{9} \mathrm{ohm}$. Therefore, the $r$ values ranging from $10^{9}$ to $10^{12} \mathrm{ohms}$ are examined in Fig. 9. The $r$ affects the $E_{s}$ significantly and therefore the radiation behavior.

\section{DISCUSSION}

\section{(A) Dopant-dependent device leakage resistance during irradiation}

It is known that the dopant in the silicon substrate will redistribute into $\mathrm{SiO}_{2}$ during oxidation. Since the dopant used in an $\operatorname{MOS}(p)$ capacitor is different from that used in an $\operatorname{MOS}(n)$ capacitor, it is expected that their oxide properties are intrinsically different. In order to show that the $r$ value during irradiation for an $\operatorname{MOS}(p)$ capacitor is smaller than that for an $\operatorname{MOS}(n)$ capacitor, the No. 1 samples in Figs $1(b)$ and 4(a) are compared. The $R C$ time constant for the 
$\operatorname{MOS}(p)$ capacitor, i.e. $6450 \mathrm{~s}$ for the curve 1 in Fig. 1(b), is larger than that for the $\operatorname{MOS}(n)$ capacitor, i.e. $2460 \mathrm{~s}$ for curve 1 in Fig. 4(a). Therefore, the $r$ value for the former one before irradiation is larger than that for the later one. But, during irradiation, the $V_{\text {is }}$ for the former one, i.e. about $-0.23 \mathrm{~V}$ for curve 1 in Fig. 2(a), is much smaller than its ideal value, i.e. $\phi_{M S}=-0.88 \mathrm{~V}$. This is quite different from that observed in the $\operatorname{MOS}(n)$ capacitor. As can be seen from Fig. $4(\mathrm{~b})$, the $V_{1 s}$ for the $\operatorname{MOS}(n)$ capacitor mentioned above is about $-0.26 \mathrm{~V}$, which is very close to its ideal value, i.e. $\phi_{M S}=-0.3 \mathrm{~V}$. According to eqn (3), the $E_{s}$ for the $\operatorname{MOS}(p)$ capacitor with a $V_{s}$ of $-0.23 \mathrm{~V}$ is about $6.8 \times 10^{4} \mathrm{~V} \mathrm{~cm}^{-1}$, while the $E_{s}$ for the $\operatorname{MOS}(n)$ capacitor with a $V_{t s}$ of $-0.26 \mathrm{~V}$ is about $4.2 \times 10^{3} \mathrm{~V} \mathrm{~cm}^{-1}$. The $\operatorname{MOS}(n)$ capacitor suffers a much smaller electric field during irradiation than the $\operatorname{MOS}(p)$ capacitor. Thus, the radiation sensitivity for the $\operatorname{MOS}(n)$ capacitor is smaller than that for the $\operatorname{MOS}(p)$ capacitor as observed in Fig. 5. From the above observations, $r$ during irradiation for the $\operatorname{MOS}(n)$ capacitor is larger than that for the $\operatorname{MOS}(p)$ capacitor according to Fig. 9. The larger the $r$ value during irradiation, the smaller the $E(t)$ during irradiation, and therefore the smaller the radiation sensitivity. The effect of $\phi_{M S}$, which determines the $E_{i}$, is only important to the initial transient ITV behavior as discussed in a later section. It is not primarily responsible for the reduction of $r$ during irradiation.

\section{(B) Transient behavior}

(1) Voltage peaks. The $V$, peaks occurred just as the $\mathrm{X}$-ray was turned on abserved in Figs 2(a), 4(b) and 6 and are mainly caused by the transient arrival of the radiation-induced electrons at the metal gates and the significant reductions of $r$ due to irradiation. When the $X$-ray is just turned on, $r$ is still quite large, the high energy electrons in the oxide layer near the metal gate will arrive at the metal gate suddenly, and induce a quick increase of $V_{r}$; but, after a short period with the X-ray on, the generation and recombination processes for the oxide trap centers may reach a stable condition, and contribute a path for the electrons accumulated on the metal gate to leak through. This change of leakage efficiency reduces $r$, and consequently decreases $V$, according to eqn (5). For an understanding of the reduction of $r$ due to irradiation, an $\operatorname{MOS}(p)$ capacitor was charged to $-5 \mathrm{~V}$ first before irradiation. Since its $R C$ time constant is quite large as mentioned earlier, the charge voltage can exist for quite a long time even when the charging source is removed from the sample. This charged sample was then irradiated by $\mathrm{X}$-rays with a dose rate of $300 \mathrm{krad} \mathrm{min}^{-1}$ for $5 \mathrm{~min}$. Its ITV behavior is shown by the dotted line in Fig. 10. It is clear from this curve that $V$, decays from $-5 \mathrm{~V}$ to about $-0.3 \mathrm{~V}$ quickly within a short period after the $\mathrm{X}$-ray is turned on; because the oxide field within the sample is negative due to the negatively charged voltage, the radiation-induced electrons will drift toward the

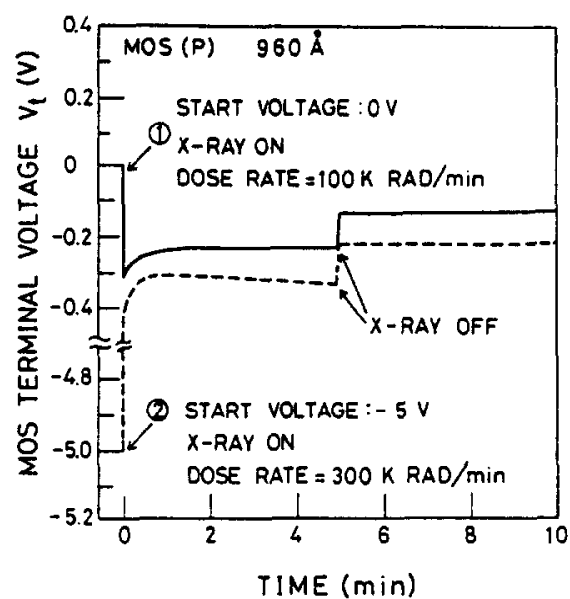

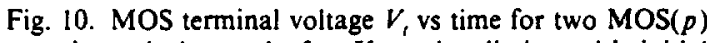
capacitors during and after $X$-ray irradiation with initial charging voltages of 0 and $-5 \mathrm{~V}$, respectively.

substrate but not the metal gate in this case. The initial charges on the metal gate (or substrate) will be reduced due to the drift of electrons. $E(t)$ is therefore reduced resulting in the decrease of $V_{1}$ as observed in this figure. However, if the $r$ value is extremely large, the drift of charges as mentioned above should be stopped when $E(t)$ is equal to zero, i.e. $V_{t}(t)=\phi_{M S}=0.88 \mathrm{~V}$, according to eqn (5). That is to say, the decrease of $V$, from $-5 \mathrm{~V}$ should not exceed $-0.88 \mathrm{~V}$ under such a circumstance. This is inconsistent with the observation that $V$, decreases to about $-0.3 \mathrm{~V}$. Therefore, there should be an extra path, i.e. the $r$ resistance, for the accumulated charges on the metal gate (or substrate) to leak through.

(2) Slowly increasing the voltage during irradiation. It is well known that the radiation effect on an MOS device is a function of irradiation time [11]. Thus, it is believed that, during irradiation, the number of the positive charges trapped near the $\mathrm{SiO}_{2} / \mathrm{Si}$ interface increases gradually with time. When these positive charges are trapped within a short distance from the $\mathrm{SiO}_{2} / \mathrm{Si}$ interface, i.e. about $20-40 \AA$ [12], the width of the energy barrier between these positive trapped charges and the $\mathrm{SiO}_{2} / \mathrm{Si}$ interface will become smaller [13], and the quantity of the hot electrons tunneling from the silicon substrate to the oxide through the energy barrier near the $\mathrm{SiO}_{2} / \mathrm{Si}$ interface will become larger. This provides a small increase to the total electron flux drifting toward the metal gate, and therefore introduces a slowly increasing $V,(t)$ as observed in all ITV measurements.

(3) Voltage drops. It is interesting to note that the voltage drops that occurred just as the $X$-ray was turned off, are very obvious for all $\operatorname{MOS}(p)$ capacitors shown in Figs 2(a), 6 and 10, but are nearly unobservable for the $\operatorname{MOS}(n)$ capacitors shown in Fig. 4(b). These observations are consistent with the previous conclusion that $r$ during irradiation for an $\operatorname{MOS}(p)$ capacitor is generally smaller than that for 
an $\operatorname{MOS}(n)$ capacitor. As can be seen from Fig. $8(b)$, there are two balanced currents flowing through the device during irradiation. One is the current induced by the drift of the radiation-induced electrons, and the other is the leakage current flowing through $r$. They are equal in magnitude, but opposite in direction, under steady-state conditions. Furthermore, the magnitude depends strongly on $r$ (or the oxide field) during irradiation. That is to say, if $r$ during irradiation is small, i.e. the $E(t)$ is large according to eqn (6), the current $I(t)$ will be large according to eqn (2). When the $\mathrm{X}$-ray is turned off, the former one will disappear since there are no radiation-induced electrons, while the latter one will still exist if and only if the charges on the metal gate (or substrate) are not equal to zero. This unbalance will cause a quick decrease of $V_{t}(t)$ until $r$ recovers to the value before irradiation. After then, $V,(t)$ will decay slowly according to the $R C$ time constant of the device. For the $\operatorname{MOS}(p)$ capacitor, its $r$ during irradiation is quite small as discussed before, so its voltage drop just as the X-ray is turned off is quite large; but, for that for the $\operatorname{MOS}(n)$ capacitor, its $r$ during irradiation is much larger than the $\operatorname{MOS}(p)$ capacitor, so that its voltage drop just as the X-ray is turned off is much smaller than for the $\operatorname{MOS}(p)$ capacitor.

\section{(C) Packaging effect}

In order to find the effect of packaging on $r$, two packaged $\operatorname{MOS}(p)$ capacitors, i.e. samples A and $B$, were examined. To start with, these samples were $R C$ decay-tested by the charge-then-decay method as mentioned in Fig. 1(a). Their $V_{l} s$ vs time are shown by curves 1 and 2 in Fig. 11, respectively. According to the estimation method mentioned in Fig. 1(b), the $r s$ of the packaged samples $A$ and $B$ are both smaller than $10^{12} \mathrm{ohm}$. Then, the bonding wires between the pins and the metal gates of these samples were removed. The $R C$ decay experiments were tested again by using a tungsten probe to directly contact the metal gate. The tungsten probe was connected to the electrometer and the charging source via a manipulator. The decay behaviors of $V_{t}$ vs time for samples $\mathrm{A}$ and $\mathrm{B}$ after

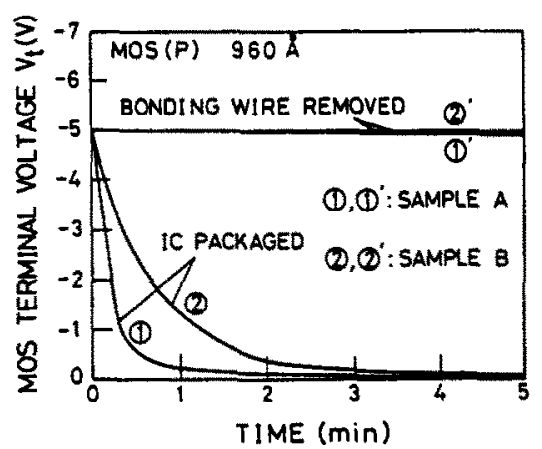

Fig. 11. The decay behavior of the MOS terminal voltage $V$, vs time for two packaged $\operatorname{MOS}(p)$ capacitors before and after removing the bonding wire. removing the bonding wires are shown by curves $1^{\prime}$ and 2 , respectively, in Fig. 11. They decay much slower than with the bonding wires present, and their $r s$ are both larger than $10^{14} \mathrm{ohm}$. Therefore, it is believed that the packaging process is important for device leakage resistance.

\section{(D) Dose rate effect}

Samples irradiated with $\mathrm{X}$-rays to the same total dose but with different dose rates will exhibit different radiation effects. Figure 12(a) shows the $V$, vs time curves for three $\operatorname{MOS}(p)$ capacitors irradiated by $\mathrm{X}$-rays to the same total dose of $500 \mathrm{krad}$ but with dose rates of 100, 50 and $10 \mathrm{krad} \mathrm{min}^{-1}$ respectively. It is to be noted that three external resistors with the same resistance of $R=10^{9} \mathrm{ohm}$ are shunted to these samples to avoid the variation effect due to different $r s$ as mentioned above. The corresponding $C-V$ curves after irradiation are shown by curves 1,2 and 3, respectively, in Fig. 12(b). The one irradiated at the smallest dose rate, i.e. curve 3, shows the largest sensitivity to irradiation. As can be seen from eqn (6), the lower the dose rate, the smaller the $\Delta n(t)$, and therefore the larger the $E(t)$. Under such circumstances, the efficiency of the hole trapping at the $\mathrm{SiO}_{2} / \mathrm{Si}$ interface is high since the separation of the radiation-induced electrons and holes is strong due to the large $E(t)$. On the contrary, a high dose rate will result in a small $E(t)$ according to eqn (6). Thus, the
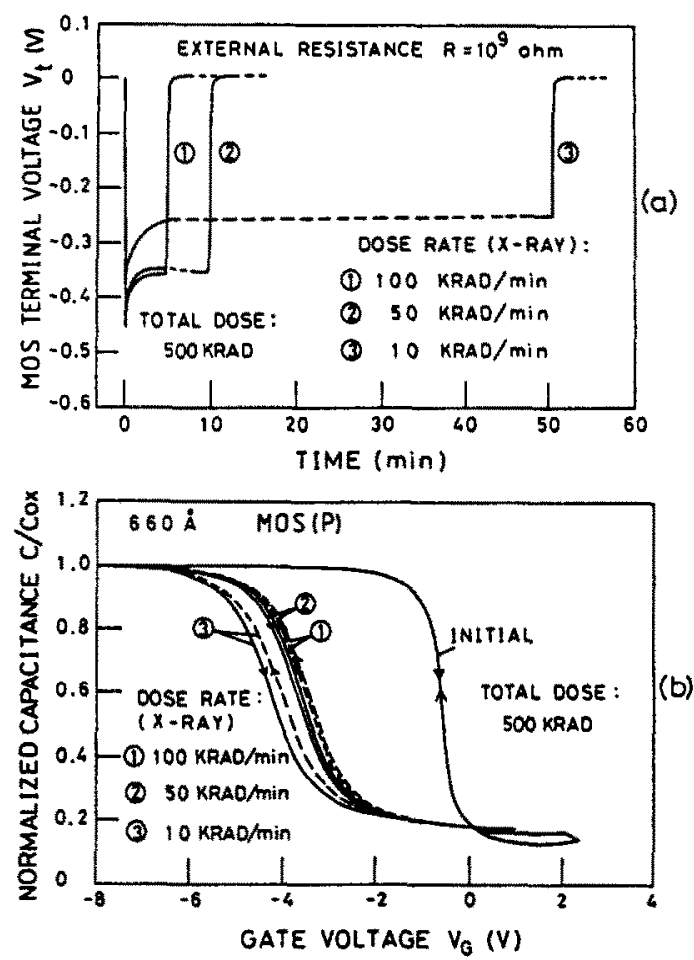

Fig. 12. (a) MOS terminal voltage $V$, vs time for three $\operatorname{MOS}(p)$ capacitors irradiated with $X$-rays with dose rates of 100.50 and $10 \mathrm{krad} \mathrm{min}^{-1}$, respectively. The total dose is $500 \mathrm{krad}$ for each $\mathrm{X}$-ray irradiation. (b) $C-V$ curves for these capacitors before and after $X$-ray irradiation. 
radiation effect on the one irradiated with a high dose rate, i.e. curve 1 , is less sensitive to irradiation than the others, i.e. curves 2 or 3 , even though their total doses are of the same magnitude. The dose rate effect is also important in determining the oxide field during irradiation.

\section{CONCLUSIONS}

The instantaneous-terminal-voltage measurement technique used in this work provides some important information about the device leakage resistance during irradiation. Using this technique, one finds that the device leakage resistance during irradiation for an $\operatorname{MOS}(p)$ capacitor is basically smaller than that for an $\operatorname{MOS}(n)$ capacitor, and that the device leakage resistance does affect efficiently the oxide field and their radiation result. The transient behavior discussed in this work gives one clearer understanding about the flow of the radiation-induced electrons within the oxide. Finally, it is suggested that the device leakage resistance effect should be taken into consideration when one is comparing the radiation effects between two samples irradiated with $X$-rays without biases.

Acknowledgements-The authors want to thank Drs W.S. Wang and S. C. Lee for their discussion, the Industrial Technology Research Institute for the supply of the X-ray irradiation source, and the National Science Council of the Republic of China for supporting this work under Contract No. NSC78-0404-E002-51.

\section{REFERENCES}

1. Nicollian E. H. and Brews J. R., MOS (Metal Oxide Semiconductor) Physics and Technology (1982) Chap. 11. Wiley, New York.

2. Schwank J. R., Winokur P. S., McWhorter P. J., Sexton F. W., Dressendorfer P. V. and Turpin D. C., IEEE Trans. Nucl. Sci. NS-31, 1434 (1984).

3. Azarewicz J. L., IEEE Trans. Nucl. Sci. NS-33, 1420 (1986).

4. Chin M. R. and Ma T. P., Appl. Phys. Lett. 42, 883 (1983).

5. Zekeriya V. and Ma T. A., Appl. Phys. Lett. 47, 54 (1985).

6. Suzuki K., Sakagami M., Nishimura I. and Watanabe K., IEEE Trans. Nucl. Sci. NS-32, 3911 (1985).

7. Dawes W. R., Fischer T. A., Huang C. C., Meyer W. J., Smith C. S., Blanchard R. A. and Fortier T. J., IEEE Trans. Nucl. Sci. NS-33, 1425 (1986).

8. Hwu J. G., Lee G. S., Lee S. C. and Wang W, S. IEEE Trans. Nucl. Sci. NS-35, 960 (1988).

9. Sze S. M., Physics of Semiconductor Devices, Chap. 7. Wiley, New York. (1981).

10. Hughes R. C., Appl. Phys. Lett. 26, 436 (1975).

11. Snow E. H., Grove A. S. and Fitzgerald D. J., Proc. IEEE 55, 1168 (1967).

12. Chang S. T. and Lyon S. A., Appl. Phys. Lett. 48, 136 (1986).

13. Chen I. C., Holland S. E. and Hu C, IEEE. Trans. Electron Devices ED-32, 413 (1985). 